\title{
Cycloaddition in Synthesis of Sulfonamide Derivatives. I. A New Method for Preparation of $N$ - $(C$-Amino- alkylthiomethylene)benzenesulfonamide
}

\author{
Tsuneo IWAKawa, ${ }^{* a}$ Hiroto Tamura, ${ }^{a}$ Tomohiro Sato ${ }^{b}$ \\ and Yoshio HaYASE ${ }^{a}$ \\ Aburahi Laboratories, Shionogi Research Laboratories, Shionogi \& Co., Ltd., ${ }^{a}$ \\ Koka-cho, Shiga 520-34, Japan and Shionogi Research Laboratories, \\ Shionogi \& Co., Ltd., Fukushima-ku, Osaka 553, Japan
}

(Received May 23, 1988)

\begin{abstract}
A novel [2+2] cycloaddition reaction of benzenesulfonyl isocyanate is reported which can serve as a new, general method for the preparation of $\mathrm{N}$ - $(\mathrm{C}$-amino-alkylthiomethylene $)$ benzenesulfonamide. Benzenesulfonyl isocyanates underwent a $[2+2]$ cycloaddition reaction with dithiocarbamates, which were obtained by treating amines with carbon disulfide and potassium carbonate, to give $\mathrm{N}$-( $\mathrm{C}$-amino-alkylthiomethylene $)$ benzenesulfonamides in good yields.
\end{abstract}

Keywords-benzenesulfonyl isocyanate; $[2+2]$ cycloaddition; dithiocarbamate; $N-(C$ amino-alkylthiomethylene)benzenesulfonamide; X-ray structure analysis

In the past decade, organic chemists have made considerable efforts to synthesize sulfonamide derivatives, because they are of interest pharmacologically. ${ }^{1)}$ However, there have been few studies on the synthesis of $N$ - $(C$-amino-alkylthiomethylene)benzenesulfonamide derivatives, in which we are interested ${ }^{2}$ : Kuwayama and Kataoka reported the synthesis of $N$-[(methylthio)aminomethylene]sulfanilamide from $N$-bis(methylthio)methylenebenzenesulfonamide, while Neidlein and Haussmann reported the preparation of $N-[C-(N-$ methylanilino)methylthiomethylene]benzenesulfonamide in three steps starting from benzenesulfonamide. In trying to obtain our target compounds, we found the former method to be of limited utility, mainly due to the low nucleophilicity of the required $N$-substituted anilines. Also, the latter method was complicated and the overall yield was unsatisfactory.

In searching for a new, more effective and convenient synthesis of our target compounds, we turned our attention to a recently discovered novel reaction of benzenesulfonyl isocyanate with dithiocarbamate which led to sulfonylimine. Here we report our new method for the synthesis of the title compounds; it is easy to perform and enables us to synthesize novel benzenesulfonamide derivatives.

Initially, the starting dithiocarbamates $(\mathbf{1} \mathbf{a}-\mathbf{f})$ were prepared from the corresponding amines and alkyl halides by a previously reported method. ${ }^{3)}$ The structures were confirmed by elemental analysis and spectral data, such as infrared (IR) and proton nuclear magnetic resonance $\left({ }^{1} \mathrm{H}-\mathrm{NMR}\right)$. The results are summarized in Table $\mathrm{I}$.

Next, reactions of these dithiocarbamates $(\mathbf{1 a - f})$ with benzenesulfonyl isocyanates were carried out. For example, la was treated with $p$-toluenesulfonyl isocyanate in toluene under reflux for $6 \mathrm{~h}$ and gave $2 \mathrm{a}$ as colorless crystals in good yield. The ${ }^{1} \mathrm{H}-\mathrm{NMR}$ spectra of $\mathbf{2 a}$ indicated the presence of methylthio protons $(\delta: 2.27,3 \mathrm{H}, \mathrm{s}), N$-methyl protons $(\delta: 3.48,3 \mathrm{H}$, s) and aromatic protons $(\delta: 6.95-8.15,10 \mathrm{H}, \mathrm{m})$. The IR spectra of $2 \mathrm{a}$ revealed a $\mathrm{C}=\mathrm{N}$ bond at $1545 \mathrm{~cm}^{-1}$. Analysis of $2 \mathrm{a}$ for carbon, hydrogen and nitrogen gave values that were in reasonably good agreement with calculated values for $\mathrm{C}_{15} \mathrm{H}_{16} \mathrm{~N}_{2} \mathrm{O}_{2} \mathrm{~S}_{2}$. On the basis of these 
TABLE I. Dithiocarbamates (1a-f)<smiles>[R15]C(=S)N([R])[R]</smiles>

\begin{tabular}{|c|c|c|c|c|c|c|c|c|}
\hline & \multirow{2}{*}{$\mathrm{R}_{1}$} & \multirow{2}{*}{$\mathbf{R}_{\mathbf{2}}$} & \multirow{2}{*}{$\mathbf{R}_{\mathbf{3}}$} & \multirow{2}{*}{$\mathrm{mp}\left({ }^{\circ} \mathrm{C}\right)$} & \multirow{2}{*}{ Formula } & \multicolumn{3}{|c|}{$\begin{array}{c}\text { Analysis ( } \% \text { ) } \\
\text { Calcd (Found) }\end{array}$} \\
\hline & & & & & & $\mathrm{C}$ & $\mathrm{H}$ & $\mathrm{N}$ \\
\hline 1a & $\mathrm{Ph}$ & $\mathrm{Me}$ & $\mathrm{Me}$ & $82-83$ & $\mathrm{C}_{9} \mathrm{H}_{11} \mathrm{NS}_{2}$ & $\begin{array}{r}54.78 \\
(54.78\end{array}$ & $\begin{array}{l}5.62 \\
5.66\end{array}$ & $\begin{array}{l}7.10 \\
7.10)\end{array}$ \\
\hline 1b & $\mathrm{Ph}$ & $\mathrm{Me}$ & $n-\operatorname{Pr}$ & $52-54$ & $\mathrm{C}_{11} \mathrm{H}_{15} \mathrm{NS}_{2}$ & $\begin{array}{r}58.62 \\
(58.71\end{array}$ & $\begin{array}{l}6.71 \\
6.71\end{array}$ & $\begin{array}{l}6.21 \\
6.24)\end{array}$ \\
\hline 1c & $\mathrm{Ph}$ & $\mathrm{Me}$ & iso- $\mathrm{Pr}$ & $72-73$ & $\mathrm{C}_{11} \mathrm{H}_{15} \mathrm{NS}_{2}$ & $\begin{array}{r}58.62 \\
(58.58\end{array}$ & $\begin{array}{l}6.71 \\
6.74\end{array}$ & $\begin{array}{l}6.21 \\
6.28)\end{array}$ \\
\hline 1d & & & $\mathrm{Me}$ & $87-88$ & $\mathrm{C}_{10} \mathrm{H}_{11} \mathrm{NS}_{2}$ & $\begin{array}{r}57.38 \\
(57.33\end{array}$ & $\begin{array}{l}5.30 \\
5.35\end{array}$ & $\begin{array}{l}6.69 \\
6.76)\end{array}$ \\
\hline 1e & $\mathrm{Ph}$ & $\mathrm{Me}$ & iso-Pent & $39-40$ & $\mathrm{C}_{13} \mathrm{H}_{19} \mathrm{NS}_{2}$ & $\begin{array}{r}61.61 \\
(61.60\end{array}$ & $\begin{array}{l}7.56 \\
7.58\end{array}$ & $\begin{array}{l}5.53 \\
5.62)\end{array}$ \\
\hline If & $\mathrm{Ph}$ & $\mathrm{Me}$ & tert $-\mathrm{Bu}$ & $108-109$ & $\mathrm{C}_{12} \mathrm{H}_{17} \mathrm{NS}_{2}$ & $\begin{array}{r}60.21 \\
(60.27\end{array}$ & $\begin{array}{l}7.16 \\
7.09\end{array}$ & $\begin{array}{l}5.85 \\
5.84)\end{array}$ \\
\hline
\end{tabular}

TABLE II. $\quad N$-( $C$-Amino-alkylthiomethylene $)$ benzenesulfonamide $(2 \mathbf{a}-\mathbf{k})$

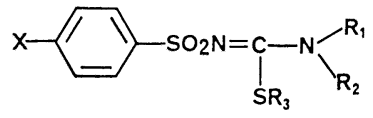

\begin{tabular}{|c|c|c|c|c|c|c|c|c|c|c|c|}
\hline & \multirow{2}{*}{$\mathrm{R}^{1}$} & \multirow{2}{*}{$\mathbf{R}^{2}$} & \multirow{2}{*}{$\mathbf{R}^{3}$} & \multirow{2}{*}{$\mathrm{X}$} & \multirow{2}{*}{$E$ or $Z$} & \multirow{2}{*}{$\begin{array}{c}\text { Yield } \\
(\%)\end{array}$} & \multirow{2}{*}{$\begin{array}{l}\mathrm{mp} \\
\left({ }^{\circ} \mathrm{C}\right)\end{array}$} & \multirow{2}{*}{ Formula } & \multicolumn{3}{|c|}{$\begin{array}{l}\text { Analysis (\%) } \\
\text { Calcd (Found) }\end{array}$} \\
\hline & & & & & & & & & $\mathrm{C}$ & $\mathbf{H}$ & $\mathbf{N}$ \\
\hline $2 \mathbf{a}$ & $\mathrm{Ph}$ & $\mathrm{Me}$ & $\mathrm{Me}$ & $\mathrm{H}$ & $Z$ & 95 & Oil & $\mathrm{C}_{15} \mathrm{H}_{16} \mathrm{~N}_{2} \mathrm{O}_{2} \mathrm{~S}_{2}$ & $\begin{array}{r}56.22 \\
(55.98\end{array}$ & $\begin{array}{l}5.03 \\
5.09\end{array}$ & $\begin{array}{l}8.74 \\
8.95)\end{array}$ \\
\hline $\mathbf{2 b}$ & $\mathrm{Ph}$ & $\mathrm{Me}$ & $\mathrm{Me}$ & $\mathrm{Cl}$ & $Z$ & 84 & Oil & $\mathrm{C}_{15} \mathrm{H}_{15} \mathrm{ClN}_{2} \mathrm{O}_{2} \mathrm{~S}_{2}$ & $\begin{array}{r}50.77 \\
(50.69\end{array}$ & $\begin{array}{l}4.26 \\
4.27\end{array}$ & $\begin{array}{l}7.89 \\
7.95)\end{array}$ \\
\hline $2 c$ & $\mathrm{Ph}$ & $\mathrm{Me}$ & $n-\operatorname{Pr}$ & $\mathrm{Me}$ & $Z$ & 90 & $105-107$ & $\mathrm{C}_{18} \mathrm{H}_{22} \mathrm{~N}_{2} \mathrm{O}_{2} \mathrm{~S}_{2}$ & $\begin{array}{r}59.64 \\
(59.50\end{array}$ & $\begin{array}{l}6.12 \\
6.23\end{array}$ & $\begin{array}{l}7.73 \\
7.77)\end{array}$ \\
\hline $2 d$ & \multicolumn{2}{|c|}{ Indolyl } & $\mathrm{Me}$ & $\mathbf{H}$ & - & 98 & $131-134$ & $\mathrm{C}_{16} \mathrm{H}_{16} \mathrm{~N}_{2} \mathrm{O}_{2} \mathrm{~S}_{2}$ & $\begin{array}{r}57.81 \\
(57.51\end{array}$ & $\begin{array}{l}4.85 \\
4.71\end{array}$ & $\begin{array}{l}8.43 \\
8.37)\end{array}$ \\
\hline $2 e$ & \multicolumn{2}{|c|}{ Indolyl } & $\mathrm{Me}$ & $\mathrm{Cl}$ & - & 82 & $161-164$ & $\mathrm{C}_{16} \mathrm{H}_{15} \mathrm{ClN}_{2} \mathrm{O}_{2} \mathrm{~S}_{2}$ & $\begin{array}{r}52.38 \\
(52.40\end{array}$ & $\begin{array}{l}4.12 \\
4.19\end{array}$ & $\begin{array}{l}7.64 \\
7.61)\end{array}$ \\
\hline $2 f$ & $\mathrm{Ph}$ & $\mathrm{Me}$ & $n$-Pr & $\mathrm{Cl}$ & $Z$ & 96 & $109-112$ & $\mathrm{C}_{17} \mathrm{H}_{19} \mathrm{ClN}_{2} \mathrm{O}_{2} \mathrm{~S}_{2}$ & $\begin{array}{r}53.32 \\
(53.30\end{array}$ & $\begin{array}{l}5.00 \\
5.05\end{array}$ & $\begin{array}{l}7.32 \\
7.26)\end{array}$ \\
\hline $2 \mathrm{~g}$ & $\mathrm{Ph}$ & $\mathrm{Me}$ & iso- $\mathrm{Pr}$ & $\mathrm{H}$ & $Z$ & 93 & Oil & $\mathrm{C}_{17} \mathrm{H}_{20} \mathrm{~N}_{2} \mathrm{O}_{2} \mathrm{~S}_{2}$ & $\begin{array}{r}58.59 \\
(58.46\end{array}$ & $\begin{array}{l}5.78 \\
5.80\end{array}$ & $\begin{array}{l}8.04 \\
7.97)\end{array}$ \\
\hline $2 \mathrm{~h}$ & $\mathrm{Ph}$ & $\mathrm{Me}$ & iso- $\mathrm{Pr}$ & $\mathrm{Me}$ & $Z$ & 89 & $83-84$ & $\mathrm{C}_{18} \mathrm{H}_{22} \mathrm{~N}_{2} \mathrm{O}_{2} \mathrm{~S}_{2}$ & $\begin{array}{r}59.64 \\
(59.51\end{array}$ & $\begin{array}{l}6.12 \\
6.18\end{array}$ & $\begin{array}{l}7.73 \\
7.72)\end{array}$ \\
\hline $2 \mathbf{i}$ & $\mathrm{Ph}$ & $\mathrm{Me}$ & iso- $\mathrm{Pr}$ & $\mathrm{Cl}$ & $Z$ & 95 & Oil & $\mathrm{C}_{17} \mathrm{H}_{19} \mathrm{ClN}_{2} \mathrm{O}_{2} \mathrm{~S}_{2}$ & $\begin{array}{r}53.32 \\
(53.32\end{array}$ & $\begin{array}{l}5.00 \\
5.07\end{array}$ & $\begin{array}{l}7.32 \\
7.32)\end{array}$ \\
\hline $2 \mathbf{j}$ & $\mathrm{Ph}$ & $\mathrm{Me}$ & iso-Pent & $\mathrm{Me}$ & $Z$ & 95 & $58-59$ & $\mathrm{C}_{20} \mathrm{H}_{26} \mathrm{~N}_{2} \mathrm{O}_{2} \mathrm{~S}_{2}$ & $\begin{array}{r}61.51 \\
(61.60\end{array}$ & $\begin{array}{l}6.71 \\
6.67\end{array}$ & $\begin{array}{l}7.17 \\
7.24)\end{array}$ \\
\hline $2 \mathbf{k}$ & $\mathrm{Ph}$ & $\mathrm{Me}$ & tert-Bu & $\mathrm{Me}$ & $E$ & 46 & $124-125$ & $\mathrm{C}_{19} \mathrm{H}_{24} \mathrm{~N}_{2} \mathrm{O}_{2} \mathrm{~S}_{2}$ & $\begin{array}{r}60.61 \\
(60.59\end{array}$ & $\begin{array}{l}6.42 \\
6.38\end{array}$ & $\begin{array}{l}7.44 \\
7.41)\end{array}$ \\
\hline
\end{tabular}



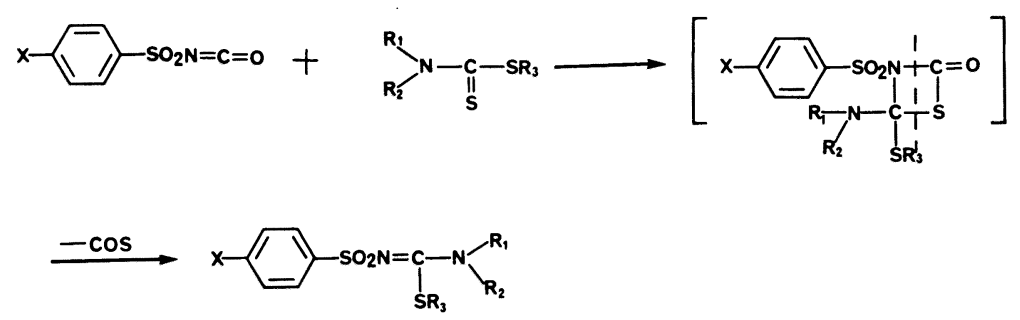

Chart 1

TABLE III. Spectral Data for Dithiocarbamates $(\mathbf{1 a}-\mathbf{f})$

\begin{tabular}{|c|c|c|}
\hline & $\begin{array}{c}\mathrm{IR} v_{\max }^{\mathrm{CHCl}_{3}} \mathrm{~cm}^{-1} \\
(v-\mathrm{N}=-\mathrm{C}=-\mathrm{S})\end{array}$ & ${ }^{1} \mathrm{H}-\mathrm{NMR}\left(\mathrm{CDCl}_{3}\right) \delta(\mathrm{ppm})$ \\
\hline 1a & 1490 & $2.55(3 \mathrm{H}, \mathrm{s}), 3.80(3 \mathrm{H}, \mathrm{s}), 6.88-7.88(5 \mathrm{H}, \mathrm{m})$ \\
\hline 1b & 1495 & $\begin{array}{l}0.92(3 \mathrm{H}, \mathrm{t}, J=9 \mathrm{~Hz}), 1.18-2.02(2 \mathrm{H}, \mathrm{m}), 3.15(2 \mathrm{H}, \mathrm{t}, J=8 \mathrm{~Hz}), 3.75(3 \mathrm{H}, \mathrm{s}), \\
6.75-7.62(5 \mathrm{H}, \mathrm{m})\end{array}$ \\
\hline 1c & 1495 & $1.33(6 \mathrm{H}, \mathrm{d}, J=7 \mathrm{~Hz}), 3.73(3 \mathrm{H}, \mathrm{s}), 4.05(1 \mathrm{H}, \mathrm{sept}, J=7 \mathrm{~Hz}), 7.07-7.57(5 \mathrm{H}, \mathrm{m})$ \\
\hline 1d & 1490 & $\begin{array}{l}2.67(3 \mathrm{H}, \mathrm{s}), 3.13(2 \mathrm{H}, \mathrm{t}, J=8 \mathrm{~Hz}), 4.42(2 \mathrm{H}, \mathrm{t}, J=8 \mathrm{~Hz}), 6.87-7.43(3 \mathrm{H}, \mathrm{m}), \\
8.77-9.10(1 \mathrm{H}, \mathrm{m})\end{array}$ \\
\hline le & 1495 & $\begin{array}{l}0.92(6 \mathrm{H}, \mathrm{d}, J=6 \mathrm{~Hz}), 2.23-2.95(3 \mathrm{H}, \mathrm{m}), 3.06-3.32(2 \mathrm{H}, \mathrm{m}), 3.75(3 \mathrm{H}, \mathrm{s}), \\
6.92-7.78(5 \mathrm{H}, \mathrm{m})\end{array}$ \\
\hline 1f & 1495 & $1.15(9 \mathrm{H}, \mathrm{s}), 2.40(3 \mathrm{H}, \mathrm{s}), 3.74(3 \mathrm{H}, \mathrm{s}), 7.10-8.05(9 \mathrm{H}, \mathrm{m})$ \\
\hline
\end{tabular}

TABLE IV. Spectral Data for $N$-(C-Amino-alkylmercaptomethylene)benzenesulfonamides (2a-k)

\begin{tabular}{lll}
\hline & \multicolumn{1}{c}{ IR $v_{\max }^{\mathrm{CHCl}_{3} \mathrm{~cm}^{-1}}$} & \multicolumn{1}{c}{${ }^{1} \mathrm{H}-\mathrm{NMR}\left(\mathrm{CDCl}_{3}\right) \delta(\mathrm{ppm})$} \\
\hline $\mathbf{2 a}$ & $1090,1145,1505,1545$ & $2.27(3 \mathrm{H}, \mathrm{s}), 3.48(3 \mathrm{H}, \mathrm{s}), 6.95-8.15(10 \mathrm{H}, \mathrm{m})$ \\
$\mathbf{2 b}$ & $1090,1245,1505,1545$ & $2.26(3 \mathrm{H}, \mathrm{s}), 3.49(3 \mathrm{H}, \mathrm{s}), 7.02-8.09(9 \mathrm{H}, \mathrm{m})$ \\
$\mathbf{2 c}$ & $1085,1140,1495,1540$ & $0.73(3 \mathrm{H}, \mathrm{t}, J=7 \mathrm{~Hz}), 0.97-1.80(2 \mathrm{H}, \mathrm{m}), 2.42(3 \mathrm{H}, \mathrm{s}), 2.70(2 \mathrm{H}, \mathrm{t}, J=8 \mathrm{~Hz})$, \\
& & $3.57(3 \mathrm{H}, \mathrm{s}), 6.95-8.27(9 \mathrm{H}, \mathrm{m})$ \\
$\mathbf{2 d}$ & $1150,1460,1480,1500$ & $2.72(3 \mathrm{H}, \mathrm{s}), 3.07(2 \mathrm{H}, \mathrm{t}, J=8 \mathrm{~Hz}), 4.25(2 \mathrm{H}, \mathrm{t}, J=8 \mathrm{~Hz}), 6.67-8.13(9 \mathrm{H}, \mathrm{m})$ \\
$\mathbf{2 e}$ & $1150,1460,1475,1500$ & $2.70(3 \mathrm{H}, \mathrm{s}), 3.10(2 \mathrm{H}, \mathrm{t}, J=8 \mathrm{~Hz}), 4.28(2 \mathrm{H}, \mathrm{t}, J=8 \mathrm{~Hz}), 6.80-8.03(8 \mathrm{H}, \mathrm{m})$ \\
$\mathbf{2 f}$ & $1090,1150,1500,1545$ & $0.75(3 \mathrm{H}, \mathrm{t}, J=7 \mathrm{~Hz}), 1.08-1.75(2 \mathrm{H}, \mathrm{m}), 2.70(2 \mathrm{H}, \mathrm{t}, J=8 \mathrm{~Hz}), 3.50(3 \mathrm{H}, \mathrm{s})$, \\
& & $7.00-8.00(9 \mathrm{H}, \mathrm{m})$ \\
$\mathbf{2 g}$ & $1085,1145,1495$ & $1.03(6 \mathrm{H}, \mathrm{d}, J=7 \mathrm{~Hz}), 3.37(1 \mathrm{H}, \mathrm{sept}, J=7 \mathrm{~Hz}), 3.53(3 \mathrm{H}, \mathrm{s}), 7.00-8.13(10 \mathrm{H}, \mathrm{m})$ \\
$\mathbf{2 h}$ & $1090,1145,1495$ & $1.10(6 \mathrm{H}, \mathrm{d}, J=7 \mathrm{~Hz}), 2.42(3 \mathrm{H}, \mathrm{s}), 3.37(1 \mathrm{H}, \mathrm{sept}, J=7 \mathrm{~Hz}), 3.53(3 \mathrm{H}, \mathrm{s})$, \\
& & $7.10-8.03(9 \mathrm{H}, \mathrm{m})$ \\
$\mathbf{2 i}$ & $1085,1145,1490$ & $1.12(6 \mathrm{H}, \mathrm{d}, J=7 \mathrm{~Hz}), 3.40(1 \mathrm{H}, \mathrm{sept}, J=7 \mathrm{~Hz}), 3.53(3 \mathrm{H}, \mathrm{s}), 7.10-8.07(9 \mathrm{H}, \mathrm{m})$ \\
$\mathbf{2 j}$ & $1090,1150,1495,1545$ & $0.72(6 \mathrm{H}, \mathrm{d}, J=6 \mathrm{~Hz}), 0.95-1.58(3 \mathrm{H}, \mathrm{m}), 2.42(3 \mathrm{H}, \mathrm{s}), 2.58-2.92(2 \mathrm{H}, \mathrm{m})$, \\
& & $3.57(3 \mathrm{H}, \mathrm{s}), 7.08-8.12(9 \mathrm{H}, \mathrm{m})$ \\
$\mathbf{2 k}$ & $1085,1145,1530$ & $1.15(9 \mathrm{H}, \mathrm{s}), 2.40(3 \mathrm{H}, \mathrm{s}), 3.74(3 \mathrm{H}, \mathrm{s}), 7.10-8.05(9 \mathrm{H}, \mathrm{m})$ \\
\hline
\end{tabular}

Sept $=$ septet

data, the structure $2 \mathrm{a}$ was established to be $N$-[C-(N-methylanilino)methylmercaptomethylene]benzenesulfonamide. In the same way, $\mathbf{2} \mathbf{b}-\mathbf{k}$ were obtained from the corresponding dithiocarbamates and sulfonyl isocyanates in good yields (Table II). These results suggest that the reaction proceeded via intermolecular $[2+2]$ cycloaddition of the $\mathrm{C}=\mathrm{N}$ group of the sulfonyl isocyanate to the $\mathrm{C}=\mathrm{S}$ group of the dithiocarbamate with subsequent loss of carbonyl sulfide.

Numerous studies have been done on the $[2+2]$ cycloaddition reaction of sulfonyl 


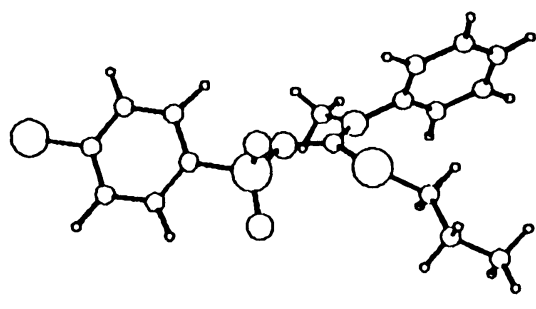

2f

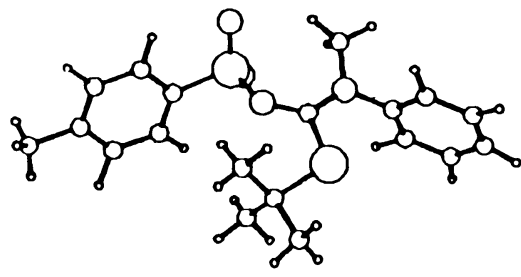

2k

Fig. 1. Molecular Structures of $\mathbf{2} \mathbf{f}$ and $\mathbf{2 k}$

isocyanates, ${ }^{4)}$ but there has been no previous report of a $[2+2]$ cycloaddition reaction of sulfonyl isocyanate with dithiocarbamate.

The configurations of the compounds obtained were assigned from their ${ }^{1} \mathrm{H}-\mathrm{NMR}$ spectra. The chemical shifts of the $N$-methyl protons could be conveniently divided into two groups: those with upfield resonance, $2 \mathbf{a}-\mathbf{c}, \mathbf{2 f}-\mathbf{j}(3.48-3.57 \mathrm{ppm})$ and that with lowfield resonance, $2 \mathbf{k}(3.74 \mathrm{ppm})$. In the ${ }^{1} \mathrm{H}-\mathrm{NMR}$ spectra of their oxime tosylates, Bull et al. found that the methine proton of the cyclopropyl group resonated at a lower field when the cyclopropyl group was cis to the sulfonyl group than when it was trans to the sulfonyl group. ${ }^{5}$ By analogy, the $E$ configuration was assigned to $2 \mathbf{k}$ which had the signal due to the $N$-methyl group at lower field, and $Z$ configurations were assigned to the others $(2 \mathbf{a}-\mathbf{c}, \mathbf{2} \mathbf{f}-\mathbf{j})$. As configurational assignments are based on relative $N$-methyl chemical shifts, $2 \mathbf{d}$ and $2 \mathbf{2 e}$ could not be assigned configurations. In order to check the ${ }^{1} \mathrm{H}-\mathrm{NMR}$ configurational assignment, $\mathrm{X}$-ray structure analyses of $\mathbf{2 f}$ and $\mathbf{2 k}$ were undertaken. The results supported our configurational assignments (Fig. 1). ${ }^{6}$

In conclusion, our new method of $[2+2]$ cycloaddition of benzenesulfonyl isocyanate provides an alternative to the known methods for the preparation of $N$ - $(C$-aminoalkylthiomethylene)benzenesulfonamide in terms of simplicity, generality and yield.

\section{Experimental}

All melting points were determined on a Yanagimoto micro melting point apparatus and are uncorrected. IR spectra were taken on a Hitachi 260-10 spectrometer. ${ }^{1} \mathrm{H}-\mathrm{NMR}$ spectra were determined on a JEOL JNM-PMX60 instrument. Chemical shifts are reported as $\delta$ values downfield relative to internal tetramethylsilane. The following abbreviations are used: $s=$ singlet, $d=$ doublet, $t=$ triplet, sept $=$ septet and $m=$ multiplet. Toluene was freshly distilled from $\mathrm{CaH}_{2}$.

General Procedure for Preparation of Dithiocarbamate $(1 \mathrm{a}-\mathbf{f})-\mathrm{K}_{2} \mathrm{CO}_{3}(34 \mathrm{mmol})$ and $\mathrm{CS}_{2}(2.6 \mathrm{ml})$ were added to a solution of the $N$-methyl amine $(30 \mathrm{mmol})$ in EtOH $(20 \mathrm{ml})$. The resulting suspension was stirred for $4 \mathrm{~h}$ at room temperature, then an alkyl halide $(30 \mathrm{mmol})$ was added dropwise and the mixture was further stirred for $20 \mathrm{~h}$. After removal of the solvent, the residue was poured into water and extracted with $\mathrm{CH}_{2} \mathrm{Cl}_{2}$. The extract was washed with water, dried over $\mathrm{Na}_{2} \mathrm{SO}_{4}$ and concentrated under reduced pressure. The residue was purified by silica gel chromatography with hexane- $\mathrm{CH}_{2} \mathrm{Cl}_{2}(1: 1)$.

General Procedure for Preparation of $\mathrm{N}$-(C-Amino-alkylthiomethylene)benzenesulfonamide (2a-k) - $\mathrm{A}$ mixture of dithiocarbamate $(4.3 \mathrm{mmol})$ and sulfonyl isocyanate $(5.2 \mathrm{mmol})$ in toluene $(11 \mathrm{ml})$ was refluxed for $6 \mathrm{~h}$. The mixture was diluted with $\mathrm{CH}_{2} \mathrm{Cl}_{2}(100 \mathrm{ml})$, washed with water $(50 \mathrm{ml})$, and dried over $\mathrm{Na}_{2} \mathrm{SO}_{4}$. After removal of the solvent, the residue was purified by silica gel chromatography with hexane-AcOEt $(2: 1)$.

Acknowledgement We wish to express our thanks to Dr. Yoshiyuki Hayashi, Director of the Aburahi Laboratories of Shionogi \& Co., Ltd., for his encouragement and permission to publish this work.

\section{References and Notes}

1) F. C. Novello and J. M. Sprague, J. Am. Chem. Soc., 79, 2028 (1957); A. A. Rubin, F. E. Roth, M. M. Winbury, J. G. Topliss, M. H. Sherlock, N. Sperber and J. Black, Science, 133, 2067 (1961). 
2) Y. Kuwayama and S. Kataoka, Yakugaku Zasshi, 85, 391 (1965); R. Neidlein and W. Haussmann, Arch. Pharm. Ber. Disch. Pharm. Ges., 300, 553 (1967).

3) W. Walter and K.-D. Bode, Angew: Chem., Int. Ed. Engl., 6, 281 (1967).

4) C. King, J. Org. Chem., 25, 352 (1960); S. Ozaki, Yuki Gosei Kagaku Kyokai Shi, 28, 17 (1970); idem, Chem. Rev., 72, 457 (1972); H. Ulrich, ibid., 65, 369 (1965).

5) M. J. Bull, J. H. Davies, R. J. G. Searle and A. C. Henry, Pestic. Sci., 11, 249 (1980).

6) The authors have deposited atomic coordinates for this structure with the Cambridge Crystallographic Data Centre. The coordinates can be obtained on request from The Director, Cambridge Crystallographic Data Centre, University Chemical Laboratory, Lensfield Road, Cambridge CB2 1EW, UK. 